\title{
Identifying Critical Isolation Valves in a Water Distribution Network: A Socio-Technical Approach
}

Noha Abdel-Mottaleb ( $\nabla$ nohaa@usf.edu )

University of South Florida https://orcid.org/0000-0002-8352-8470

Payman Ghasemi Saghand

University of South Florida

Mathews Wakhungu

University of South Florida

Hadi Charkhgard

University of South Florida

E. Christian Wells

University of South Florida

Qiong Zhang

University of South Florida

\section{Research Article}

Keywords: isolation valves, criticality , water distribution networks , network analysis

Posted Date: June 4th, 2021

DOI: https://doi.org/10.21203/rs.3.rs-416140/v1

License: (c) (i) This work is licensed under a Creative Commons Attribution 4.0 International License.

Read Full License 


\title{
1 Identifying Critical Isolation Valves in a Water
}

\section{Distribution Network: A Socio-Technical Approach}

\author{
3 Noha Abdel-Mottaleb • Payman Ghasemi \\ 4 Saghand - Mathews Wakhungo - Hadi \\ 5 Charkhgard · E. Christian Wells · Qiong \\ 6 Zhang* \\ 7 Received: date / Accepted: date
}

8 Abstract Isolation valves are critical for reliable functioning of water distri-

9 bution networks (WDNs). However, it is challenging for utilities to prioritize

10 valve rehabilitation and replacement given it is often unclear if certain valves

\footnotetext{
N. Abdel-Mottaleb

University of South Florida

Tampa, FL USA 33620

E-mail: nohaa@usf.edu

P. Saghand

University of South Florida

Tampa, FL USA 33620

E-mail: payman@usf.edu

M. Wakhungu

University of South Florida

Tampa, FL USA 33620

E-mail: mwakhungu@usf.edu

H. Charkhgard

University of South Florida

Tampa, FL USA 33620

E-mail: hcharkhgard@usf.edu

E C. Wells

University of South Florida

Tampa, FL USA 33620

E-mail: ecwells@usf.edu

Q. Zhang *Corresponding Author

University of South Florida

Tampa, FL USA 33620

E-mail: qiongzhang@usf.edu
} 
11

12

$13 \quad$

14

15

16

17

30 While many stressors act on water distribution networks (WDNs) (e.g., cli31 mate change, aging infrastructure, interdependencies), utilities have limited 32 resources to address challenges WDN infrastructure faces. This makes priorзз itizing WDN components (e.g., pipes, valves, pumps, tanks) to repair, reha34 bilitate, or replace important. Much research has focused on the prioritization 35 of pipes, based on pipe criticality (Shuang et al., 2014; He and Yuan, 2019; 36 Abdel-Mottaleb et al., 2019). The criticality of pipes is determined using sev- 
37 eral indicators such as system demand shortfall. The major assumption is that

38 a single pipe can be isolated for repair. However, the minimum isolatable unit

39 of WDNs is a segment, and segments often consist of more than a single pipe.

40 This is because in real world WDNs, isolation valves are not available at the 41 end of each pipe (Giustolisi and Savic, 2010; Santonastaso et al., 2019).

When pipes are to be accessed for repair, rehabilitation, or replacement, 43 isolation valves must be operated to isolate segments of the WDNs (Liu et al., 44 2017). In the extreme case that a network does not have sufficient operable 45 isolation valves, the entire network would need to be isolated from its water 46 sources for any pipe repair (i.e., every customer's water service is disrupted). 47 In fact, this has occurred recently, in 2013, in a town in Oklahoma, USA 48 (WaterWorld, 2014). Though this is an extreme case, it often happens that 49 more segments must be isolated because of inoperable valves-leaving more 50 customers without water, some of which are potentially more vulnerable than ${ }_{51}$ others to the water loss. In addition, valves may become inoperable unless ${ }_{52}$ they are exercised at regular time intervals at least once a year; more often, 53 for abrasive water (Mays et al., 2000; Jun et al., 2007).

Prioritizing valves is more complicated than the common assumption of 55 single pipe isolation, because a single valve can be responsible for the isolation 56 of many segments (depending on the given scenario of valve operability in the 57 network). Valves are critical when the segments or zones they help prevent 58 from unintended isolation are important/critical themselves. The importance 59 of segments and valves has been addressed in the literature in two different 60 ways: analysis and design (Jun et al., 2007). Design is concerned with opti61 mal placement of valves ( e.g., Giustolisi and Savic (2010); Creaco and Haidar 62 (2019); Giustolisi (2020); Santonastaso et al. (2019)). Analysis involves evalu63 ating the impacts of valve failure (or inoperability) on WDN performance and 64 prioritizing valves and segments to inform operation and management. There 
${ }_{87}$ each inoperable valve; however, there can be many combinations of inoperable

88 valves in real WDNs. In addition, because there are often loops of segments

s9 in real WDNs, there can be many paths between one segment and another 90 (i.e., that need to be isolated). It is resource intensive to enumerate all pos${ }_{91}$ sible scenarios for utilities. Mahmoud et al. (2018) prioritized repair actions ${ }_{92}$ (including operating valves) using multi-objective optimization; where the ob- 
jectives were the minimization of the negative impact on the consumers (e.g., unsupplied water volume) and the minimization of the corresponding number of repair actions. The purpose of their method is real-time decision making after failures assuming valves are operable.

Furthermore, all of these previous studies do not account for the social vulnerability of communities serviced by the WDN (i.e., customers vulnerable to a disruption in water service). In reality, utilities are often concerned with social indicators to varying degrees and account for it within the score of consequences of failure in their risk-based asset management. In addition, previous studies do not account for uncertainties associated with both water service needs of customers and customer vulnerability (Shuang et al., 2019), which are inherent in infrastructure networks and the urban environment (Walski, 2001; Zischg et al., 2018). Accounting for these uncertainties has been shown to have an effect on resulting best practices or decisions (Diao et al., 2019). Finally, the likelihood of failure of segments has not been included in these studies (Giustolisi, 2020), but considering it can provide utilities with insight on the valves that ought to be repaired sooner. In sum, challenges to prioritizing isolation valves include the complexity of accounting for multiple possible operable valve scenarios in a network, and accounting for social vulnerability regarding the service population, uncertainties in both hydraulic performance and social vulnerability, and the likelihood of segment failure. These limitations can be attributed to the methodologies used for analyzing WDN valving.

To address these limitations, this study develops a methodology and applies it to the City of Tampa as a case study. The presented methodology is geared towards long-term planning as opposed to real-time operation, to provide a prioritization of valves to rehabilitate, repair or replace while considering the potential inoperability of valves. Graph theory is combined with multi-criteria optimization to identify the pareto-optimal sets of critical valves 
121

(i.e., valves which have the most potential adverse impact when inoperable) based on hydraulic performance and social vulnerability of WDN segments. In lieu of a priori knowledge on valve operability, the deterministic model identifies critical valves and the results are compared with models that account for both uncertainties and likelihood of failure in segments for the City of Tampa.

\section{Methods}

To identify critical valves, an optimization model is used over the logical implications of inoperable valves. The implications of inoperable valves are segment isolation and the potential subsequent loss of water service. Not all segment isolation has the same effect-some segment isolation is more critical than others. To obtain the logical implications, first a segment-valve representation of the network is constructed. Then, the segment-valve representation is simplified to a gomory-hu tree network model (i.e., an equivalent flow graph), that allows identification of the minimum set of inoperable valves that would cause any given segment isolation.

\subsection{Segment-Valve Representation}

The segment-valve representation of the WDN has often been called the mathematical "dual" of the more common pipe-junction representation. In this representation, segments containing pipes are the nodes and isolation valves are the edges, or links, between them. To obtain the segment-valve representation, a hydraulic network model based on pipe-junction representation is first created using a hydraulic modeling software (e.g., WaterGEMS). The data structure (e.g., list of lists, dictionary) containing segments and associated isolation valves is exported from the software (e.g., WaterGEMS) to python. 
A network model of segments and valves is then generated using the networkx package (Abdel-Mottaleb and Walski, 2020).

There are still loops in the segment-valve representation, making it computationally complex to identify how valves impact segments when any valves are inoperable. This is because there are many potential combinations of valves that can impact isolation of a given segment. Consider the small network shown in Figure 1a, where node 1 (i.e., segment 1) contains the reservoir and node 6 (i.e., segment 6) contains a tank. In this figure, if segment 4 must be isolated, at least two operational valves must also exist (one on each path) between segment 2 and segment 4 , so that segment 2 is not isolated unnecessarily. The combinations of two operable valves that allow segment 4 to be isolated without isolating segment 2 correspond to the minimum k-cut between segments 2 and 4 , where $k=2$. A minimum k-cut between two nodes (i.e., segments) means that $\mathrm{k}$ edges (i.e., valves) are required to partition the two nodes (i.e., successfully isolate them from each other). If the valves between segments 2 and 4 , and segments 4 and 5 are both inoperable, then to isolate segment 4 , the entire network must also be isolated from the reservoir. This illustrates the combinatorial complexity of assessing the impact of failed valves on segments because of the existence of loops of segments.

\subsection{Gomory-Hu Tree of Segment Connectivity to Address Combinatorial Complexity}

The gomory-hu tree (G-H tree for short) simplifies the task of identifying the paths along which operational valves should exist to minimize unnecessary segment isolation (i.e., the minimum k-cuts between segments). When there are loops of segments, a set of valves instead of a single valve must be operational to minimize unnecessary segment isolation. The G-H tree contains 
nodes, which represent segments, and edges linking segments that represent the flow paths between each pair of connected segments. Specifically, edges represent the $\mathrm{k}$ valves (corresponding to all the minimum $\mathrm{k}$-cut(s) in the segment valve network, or the minimum number of valves that must be operable) between two connected nodes for successful isolation. The G-H tree is also called a flow equivalent graph, because each minimum cut(s) represents all of the possible flow paths between two nodes (i.e., segments). The G-H tree of the small example in Figure 1b. In this figure, the edge from segment 2 to segment 3 represents the following valves from Figure 1a: segment 2-segment 3, and either segment 2-segment 4, segment 4-segment 5, or segment 5-segment 3. The G-H tree of the segment valve representation of the network is obtained using the gomory_hu_tree function within networkx in python. The valves that are part of the minimum cut(s) are stored in a data structure for the following step.

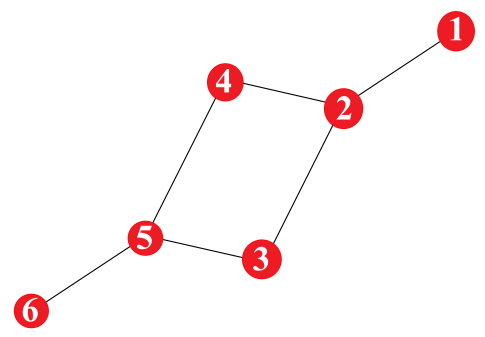

(a) segment-valve representation of a small example network

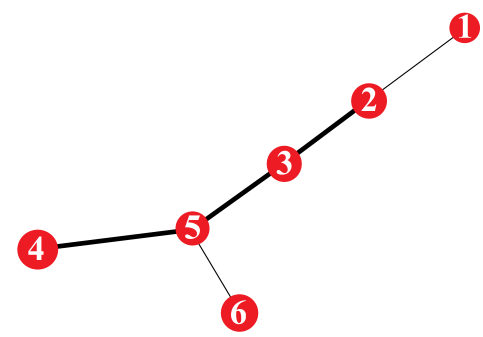

(b) Gomory-Hu tree of small example, where edges that represent more than one valve are bolded

Fig. 1: Segment-valve represenation and Gomory-Hu tree of a network example 
2.3 Logical Network

A logical network is constructed to represent the implications of valve failure on segments outage following a similar methodology to that presented in AbdelMottaleb et al. (2019). In this work, valve failure refers to valves that are in a failed (OPEN) state. In the logical network, there are two types of nodes. The nodes represent either segments (see the red nodes in Figure 2) or sets of valves (i.e., edge of G-H tree or minimum cut in the original network) (see the blue nodes in Figure 2). The edges in the logical network are directed and represent a logical implication that a given set of valves will have an adverse consequence on connected segments. For example, if the valve(s) represented by node 1-2 in Figure 2 fails, it will have adverse consequence on segments 1 and 2 . If one of the $\mathrm{k}$ valves in a minimum cut between two segments is inoperable, then the segment it is connected to will have to be isolated in order to isolate adjacent segments (due to the valve failure). Figure 2 illustrates the construction of the logical network for the small example illustrated in Figure 1. If segments are identified as critical in the optimization, then the valves connected to them are also identified as critical. For example, if segments 2 and 5 are critical, 2-3, $2-4,3-5,5-4$ and $5-6$ are part of the solutions set.

\subsection{Multi-Criteria Optimization}

A multi-criteria optimization model is developed to identify the critical valves based on their adverse impact to segments. The logical network relates valves to segments and is used as input for the optimization so that the impact of segment isolation can be quantified and the set of inoperable valves that would lead to the worst segment isolation scenarios can be subsequently identified. The objective functions are quantified for each node representing valves in the logical network by aggregating the attribute value for the segments impacted 


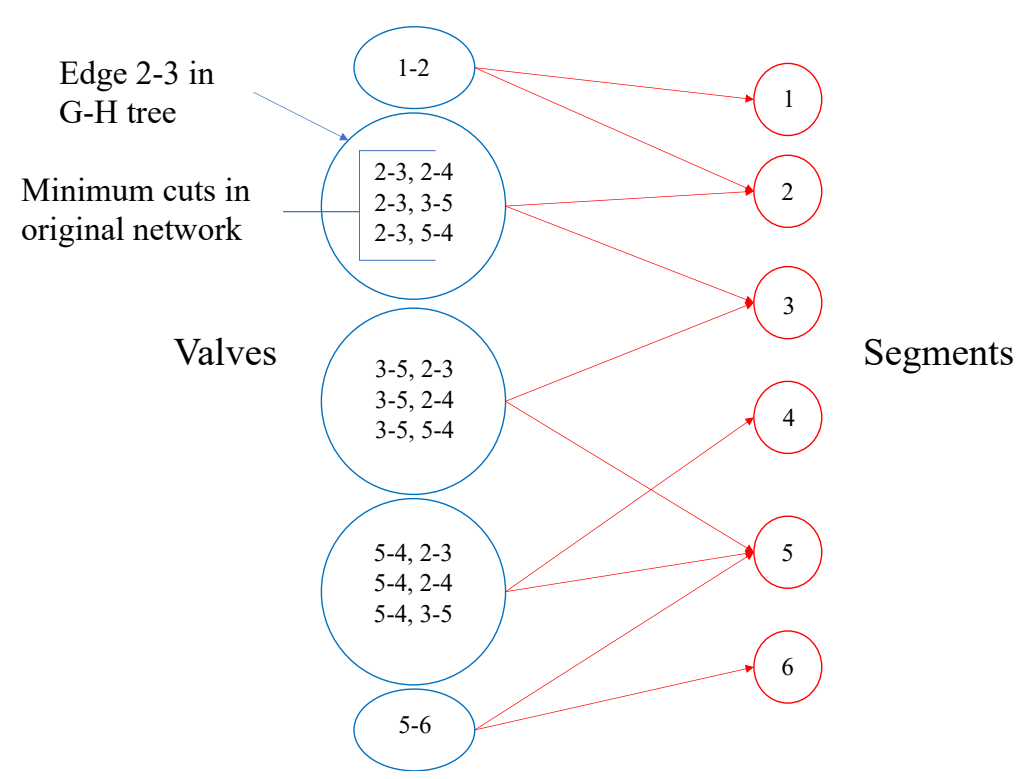

Fig. 2: representation of the logical network, where valves that were represented by edges are modeled as nodes and segments are also modeled as nodes, and the edges between valves and segments represent logical implications

by a selected valve or set of valves. Three objective functions (i.e., criteria) are used to quantify the impact to segments: reachability, segment flow volume, and social vulnerability. The attribute values for each segment under each criterion are calculated and stored as a dictionary object.

Reachability refers to how reachable the water source(s) is to other segments after a given segment isolation. In this study, reachability is quantified using the importance index that is calculated from a matrix built from the segment-valve representation of the WDN as described in Abdel-Mottaleb and Walski (2020). The matrix contains rows $(m)$ representing isolated segments and columns $(n)$ representing affected segments, where for each cell $(m, n)$ a value of 2 is assigned if the isolation of segment $m_{i}$ causes segment $n_{j}$ to be isolated from all sources; a value of 1 is assigned if the isolation of segment $m_{i}$ causes segment $n_{j}$ to be isolated from the reservoir but remain 
connected to tanks; a value of 0 is assigned if segment $n_{j}$ remains connected

to the reservoir. The summation of each row is the importance index of the segment represented by that row. The importance index of a segment calculated from the matrix is an indicator of the system demand shortfall for that given segment isolation, and more specifically, the number of segments that lose connection with water sources. In this manner, a segment with a high value for reachability (as measured by the importance index) is a segment that would cause many other segments to be disconnected from water sources. This objective function can be substituted with simulation data (e.g., demand shortfalls under segment isolation scenarios). In this study, reachability and segment flow volume are used separately to uncouple the topology from the hydraulics in the analysis and to compare the identified critical valves obtained with and without inclusion of additional objectives. Reachability can be used to identify critical segments, and subsequently valves in two ways, graph theory and a single objective optimization by only including the objective function related to reachability. Graph theory can be used by applying the articulation_points function within networkx package in python over the segment-valve representation, as demonstrated in Abdel-Mottaleb and Walski (2021).

Segment flow volume is the second objective function used to assess the impact from valves to segments. The water flow volume in a segment is calculated using WaterGEMs ((Bentley Systems, 2020) through pressure-driven extended period simulation, over a 24-hour duration. In other case studies, it may be appropriate to substitute these two objective functions (reachability and segment flow volume) with just one using the system demand shortfall obtained from hydraulic simulations. The flow volume through segments varies from the initial design of a WDN and varies over time as customer demands change. It has been shown that larger pipes, holding more flow, have a larger 
adverse impact on WDNs than smaller counterparts (Paez et al., 2020). Therefore, larger mains are typically designed with a longer design life than smaller pipes because of their high cost and have lower uncertainties in flow volume. In this study, it is assumed that uncertainties in flow volume increase as pipe diameters decrease.

A novelty presented in this work is accounting for segments with populations that are vulnerable to water loss given their social class, household composition, sensitive population, minority, housing tenure, and quality of life (see Borden et al. (2007); Cutter et al. (2003)). The third objective function, therefore, accounts for the social vulnerability of populations associated with each segment . The social vulnerability index (SVI) used in this study was developed by Wakhungu (2020). The index was calculated using 2016 and 2017 sociodemographic data obtained from the American Community Survey and the U.S. Census Bureau. Using R-mode factor analysis in SPSS v. 25, the study considered 14 social vulnerability indicators to derive a SVI for each census block group in the City of Tampa. The analysis involved weighting component scores by the percentage of variance explained and aggregating the scores into a cumulative factor score indicating the vulnerability of a census block group. The social vulnerability of a community with a high SVI is also more likely to change than a community with a low SVI because of gentrification occurring in Tampa (See Chavez (2013); Wakhungu (2020). In other words, there is more uncertainty associated with high SVI values than with low SVI values. Previous criticality analyses using the pipe-junction representation have taken key customer, or important demand junctions into account (e.g., Giustolisi (2020)). Similarly, the social vulnerability index accounts for various household composition and sensitive population variables per census block group including the elderly, infants and children, people living with a disability or illness, and large households. 


\subsubsection{Formulation}

In this study, three level of formulations are used to solve the problem accounting for different aspects. However, the overall formulation requires the decision-maker to provide the number of critical sets of valves that the model should identify. In other words, for a given user-defined number, the model looks for the worst sets of valves to fail in the network.

Formulation One The first formulation is the most straightforward one and is based on the two following assumptions. First, we assume that there does not exist any uncertainty in the objective functions over time or in their measurement. The second assumption is that the likelihood is the same for all valves to be operational (i.e., all segments have an equal likelihood of failure).

To mathematically formulate the model, we define $S$ as the set of all segments, and $s_{i}$ as the binary decision variable related to segment $i \in S$, where $s_{i}=1$ means that segment $i$ must be isolated and $s_{i}=0$ means otherwise. Also, we let $V$ be the set of all valves, and $v_{j}$ represent the binary decision variable related to valve $j \in V$, where $v_{j}=1$ means that valve is inoperable and $v_{j}=0$ means otherwise. Based on the logical network, $V_{i}$ is defined as the set of all the valves affecting the segment $i$ (i.e., must be operational such that segment $i$ is not unnecessarily isolated) such that $\bigcup_{i=S} V_{i}=V$. In addition, we define $\boldsymbol{a}:=\left(a_{1}, a_{2}, \cdots, a_{|S|}\right), \boldsymbol{b}:=\left(b_{1}, b_{2}, \cdots, b_{|S|}\right)$, and $\boldsymbol{c}:=\left(c_{1}, c_{2}, \cdots, c_{|S|}\right)$ as the vectors of non-negative real numbers where $a_{i}, b_{i}$, and $c_{i}$ are the coefficients representing the social vulnerability, segment flow volume, and reachability impact of segment $i \in S$, respectively.

The optimization model includes three objective functions to be maximized for identifying the worst sets segments to be isolated. Subsequently, from the relationship between segments and valves in the logical network, the worst sets of valves to be inoperable are identified by selecting the sets of valves connected 
307 to the identified segments in the logical network. The objective functions of 308 our optimization model are as below:

309 - Social Vulnerability:

$$
\max \sum_{i \in S} a_{i} s_{i}
$$

310 - Segment Flow Volume:

$$
\max \sum_{i \in S} b_{i} s_{i}
$$

311 - Reachability Impact:

$$
\max \sum_{i \in S} c_{i} s_{i}
$$

The constraints of the optimization model are as follows:

$$
\begin{array}{ll}
s_{i} \leq \sum_{j \in V_{i}} v_{j} & \forall i \in S, \\
\left|V_{i}\right| s_{i} \geq \sum_{j \in V_{i}} v_{j} & \forall i \in S, \\
\sum_{i \in S} \sum_{j \in V_{i}} v_{j}=k & , \\
v_{j} \in\{0,1\} & \forall j \in V, \\
s_{i} \in\{0,1\} & \forall i \in S .
\end{array}
$$

\footnotetext{
312 Constraints (4) and (5) define the relationship between valves and seg313 ments such that, if and only if at least one of the valves affecting segment $i$ is 314 inoperable (from the minimum set of valves that must be operable), then seg315 ment $i$ must be unnecessarily isolated. Note that, since all the objective func316 tions of our optimization problem are in maximization form, Constraint (5) 317 will be naturally satisfied, and therefore, can be removed from the model. Con318 straint (6) defines the user-imposed condition on the total number of valves to fail, where $k$ is a positive integer number. Constraints (7) and (8) enforce
} 


$$
\sum_{i \in S} s_{i}=k
$$

325 Proof. Since, $\left|V_{i}\right|=1$ by assumption, we can rewrite Constraints (4) and (5) 326 as follows:

$$
\left.\begin{array}{c}
s_{i} \leq \sum_{j \in V_{i}} v_{j} \quad \leftrightarrow \quad s_{i} \leq v_{i} \\
\left|V_{i}\right| s_{i} \geq \sum_{j \in V_{i}} v_{j} \quad \leftrightarrow \quad s_{i} \geq v_{i}
\end{array}\right\} \quad \rightarrow \quad s_{i}=v_{i}
$$

where $v_{i}$ is the binary decision variable showing whether the valve affecting the segment $i$ is inoperable or not. This combined with Constraint (6) results in

$$
\sum_{i \in S} \sum_{j \in V_{i}} v_{j}=k \quad \leftrightarrow \quad \sum_{i \in S} v_{i}=k \quad \leftrightarrow \quad \sum_{i \in S} s_{i}=k
$$

Formulation Two In this formulation, the model accounts for the uncertainties that are inherent in two of the objective functions of the study. Uncertainties in segment flow volume are assumed to increase (between 5 percent and 95 percent of a given flow value, evenly distributed based on quantile) as pipe diameters decrease. Uncertainties in social vulnerability are assumed to increase as the social vulnerability index increases (also between 5 percent and 95 percent of a social vulnerability index value, evenly distributed based on quantile). This assumption is specific to the case study and may differ for other cities. 
352 and segment flow volume impact are defined as follows:

353 - Social Vulnerability:

$$
\max \sum_{i \in S} \underline{a_{i}} s_{i}+\left(\overline{a_{i}}-\underline{a_{i}}\right) r_{i}
$$

354 - Segment Flow Volume:

$$
\max \sum_{i \in S} \underline{b_{i}} s_{i}+\left(\overline{b_{i}}-\underline{b_{i}}\right) r_{i}
$$

where $r_{i}$ is a binary variable that shows whether the worst-case is happening for segment $i$ or not. The constraints of the robust optimization model include the constraints of formulation one, i.e., constraints (4)-(8), in addition to the 
following constraints:

$$
\begin{array}{ll}
r_{i} \leq s_{i} & \forall i \in S, \\
\sum_{i \in S} r_{i} \leq \gamma\left(\sum_{i \in S} s_{i}\right) & \forall i \in S .
\end{array}
$$

Formulation Three This formulation is the most realistic model that accounts for both the uncertainties that are inherent in two of the objective functions of this study as well as the likelihood of failure within segments (based on pipe failure within the segments). Though the likelihood of failure is complex and there are models that have been developed accounting for pipe length, age, size, and material among many other things. In this work, we use age as the primary factor because most of the pipes in the City of Tampa are severely aged (Park et al., 2010).

Based on the likelihood of failures in a given segment, we categorize the set of valves into three categories shown by $V^{h}, V^{m}$, and $V^{l}$ representing the sets of valves affecting segments (i.e., the valves that successfully isolate given segments) with high, medium, and low likelihood of need to be operated, respectively. Following this definition, we simply substitute constraint (6) with the following set of constraints:

$$
\begin{aligned}
\sum_{j \in V^{h}} v_{j} & =h, \\
\sum_{j \in V^{m}} v_{j} & =m, \\
\sum_{j \in V^{l}} v_{j} & =l .
\end{aligned}
$$


These constraints impose the user-defined condition on the total number of valves to fail. In these constraints, $h, m$, and $l$ are the user-defined nonnegative integer numbers that represent the number of failures in different categories based on their likelihood of needing to be operated. Note that, as $V^{h}, V^{m}$, and $V^{l}$ have high, medium, and low likelihood of needing to be operated, respectively, their corresponding user-defined conditions should be defined such that $l \leq m \leq h$ and $h+m+l=k$.

\subsubsection{Solution Approach}

The formulations defined in this study represent a multi-objective optimization problem with three conflicting objectives, or criteria: reachability, segment flow, and social vulnerability. The inclusion of three different objectives in the model makes it impossible to find a single solution that simultaneously optimizes all the objectives. Therefore, we focus on finding the set of solutions for which it is impossible to increase one objective without decreasing other objectives. Such solutions are referred to as efficient or non-dominated solutions in the literature of multi-objective optimization (Saghand et al., 2019).

The importance of finding different efficient solutions is that they help the decision-maker better understand the trade-offs between the objective functions. Therefore, in this study, we only focused on approaches that can generate the entire set of efficient solutions of our problem. Specifically, we implemented two different algorithms, developed by Dächert and Klamroth (2015) and Boland et al. (2017) in C++ using Gurobi Optimizer V9.0.3 and tested their performance on our specific problem. Among the mentioned algorithms, we chose the method developed by Boland et al. (2017), referred to as the "quadrant shrinking method" as it resulted in the best performance in terms of time and computational complexity. Finally, we note that the computational experiments were conducted on a Dell PowerEdge R360 with two Intel 
Xeon E5-2650 2.2 GHz 12-Core Processors (30MB), 128GB RAM, the RedHat

Enterprise Linux 6.8 operating system, and using Gurobi's default setting.

407 The model was applied to a field validated, skeletonized WDN model from the 408 City of Tampa. Tampa's WDN has over 134,000 pipes serving over 600,000 409 customers (Park et al., 2010). There is a single reservoir and 5 tanks through410 out the network. The network has approximately 50,000 isolation valves, many 411 of which are inoperable. Therefore, the city is prioritizing valve rehab and re412 placement-providing impetus for this study. This study tested $k=10, k=20$, ${ }_{413}$ and $k=30$ and $k=30$ is selected because it is the most representative. In 414 this study, $\gamma$ was assigned a value of 0.5 and $h, m$, and $l$ are assigned values 415 of 15,9 and 6 , respectively. 
 \\ 4 Results and Discussion}

417

\subsection{Spatial Variation of Segment Attributes}

Prior to applying the developed optimization models, critical WDN locations are mapped based on each segment attribute separately. Segment reachability is mapped in Figure 3a, where segments bridging between water sources and more peripheral locations of the network are shown to have higher values. In Figure 3b, valves identified as critical using graph theory based on the reachability to the source are mapped. Only 22 valves are identified using this method, and the identified valves are clustered in 6 different locations in the city, circled in Figure 3b. In the South-West of the city, where there are three different storage tanks, no critical valves are identified using reachability alone. Clusters 1 and 6 are located in Northeast and Southeast Tampa, respectively. The valves in these locations are selected due to a lack of redundancy in water pipes. The clusters are locations that bridge between the water reservoir and more remote water distribution segments. The flow volume of segments is mapped in Figure 4. There are few segments in the highest percentile of flow volume, and they correspond to the distribution mains, that ensure there is sufficient volume being transported from the water source(s) to the sparser periphery of the network. It is interesting to note that the Central-South of the City (corresponding to Downtown) has many segments with relatively low flow volume. This is likely because of the high redundancy in this part of the City. In contrast, in Sulphur Springs and Temple Terrace, near the water reservoir, the pipes are generally larger to convey flow to the rest of the network. It is interesting to note that these communities are socially vulnerable, as shown in Figure 5. Many of the identified critical valves, shown in Figure 3b, also coincide with socially vulnerable communities. In Tampa, vulnerable and non- 


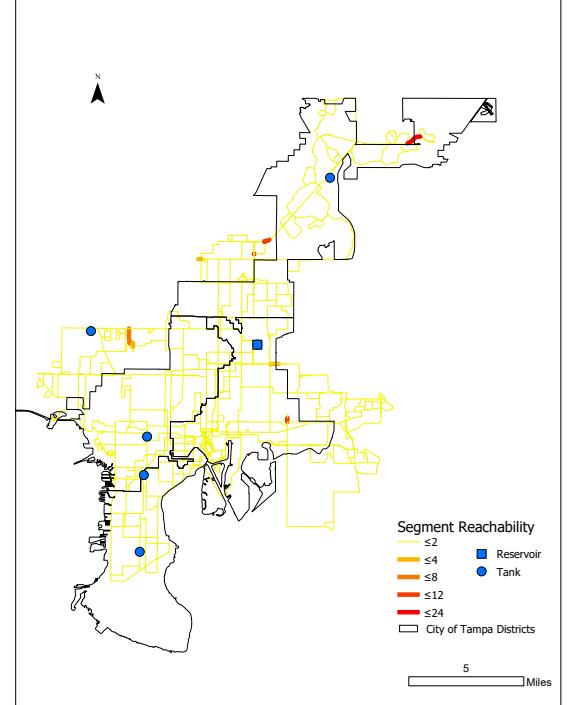

(a) Map of segment reachability values

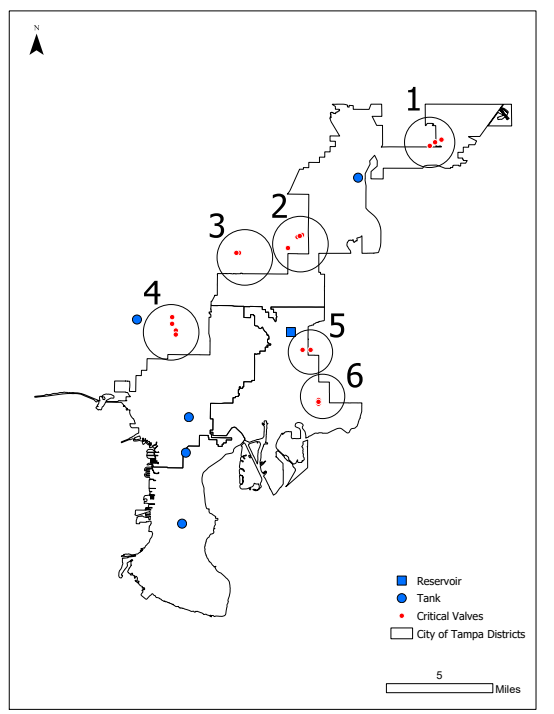

(b) Map of critical valves

Fig. 3: Segment reachability values and the subsequently identified critical valves

vulnerable neighborhoods are not completely isolated from each other. Figure 5

shows pockets of lower vulnerability blocks interspersed among neighborhoods with high vulnerability. However, there is generally less vulnerability in the Southwest and Northeast of the city, in comparison with the Center. Interstate 275 (running approximately North-South) intersects the center of the city, dividing communities and highlights where there are many pockets of socially vulnerable communities. An interesting observation is that because many of the communities that are socially vulnerable in the city have been historically marginalized, they coincide with aging pipes and thus high likelihood of failure in the water distribution segments. However, there are also a few communities with historically very low social vulnerability, yet having aging pipes as well, in the South of the city as shown in Figure 6. By mapping the segment attributes (reachability, segment flow volume, and social vulnerability) individually, it is 


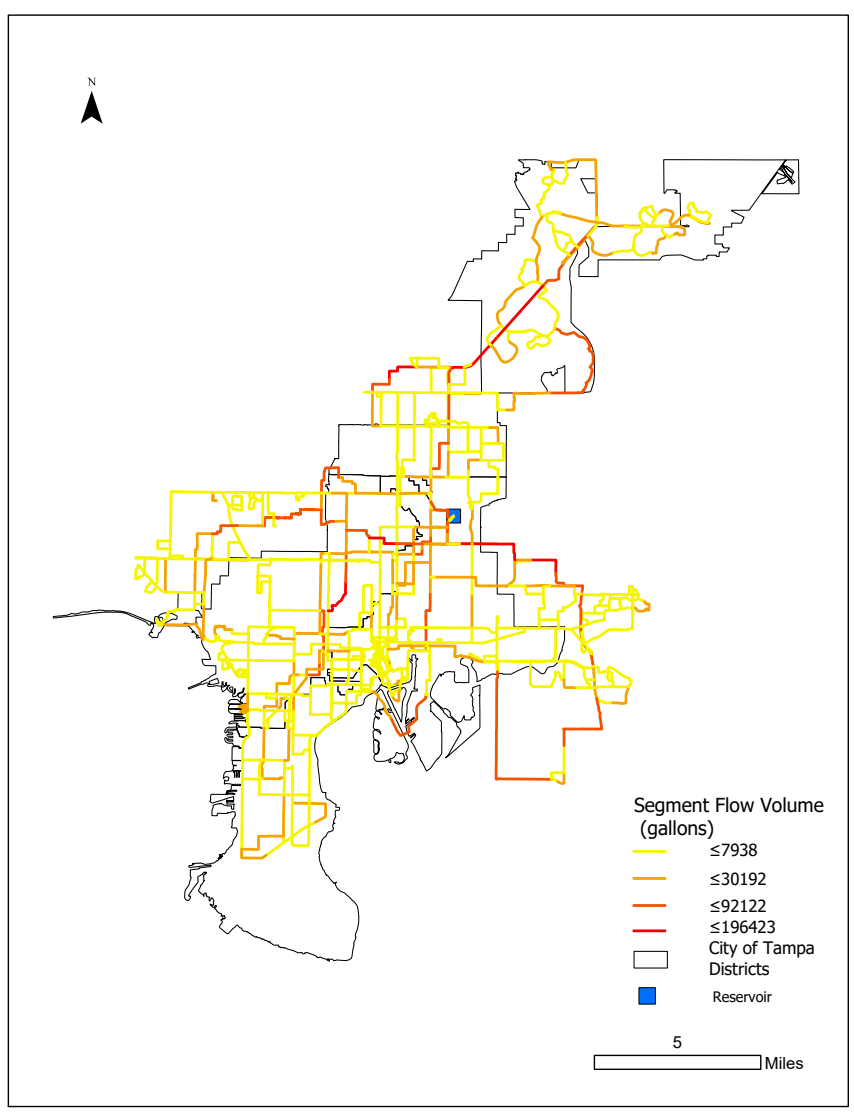

Fig. 4: Map of segment flow volume

455 clear that there exist conflicting locations of criticality. For example, in the ${ }_{456}$ West of the City (near cluster 4 in Figure 3b), segments have high reachability ${ }_{457}$ values and high social vulnerability, but they are not severely aged, nor do they ${ }_{458}$ have high flow volume relative to other segments. Likewise, in the downtown 459 area in the South-Central location of the city, segment flow volume is relatively 460 low due to the high level of redundancy; however, there are segments with 461 highly aging pipes and high social vulnerability. Additionally, segment age, 462 which is used as a surrogate for the likelihood of failure, follows a different 463 spatial pattern (generally lower age for locations further away from downtown) ${ }_{464}$ than the three segment attributes. Therefore, optimization models are useful 


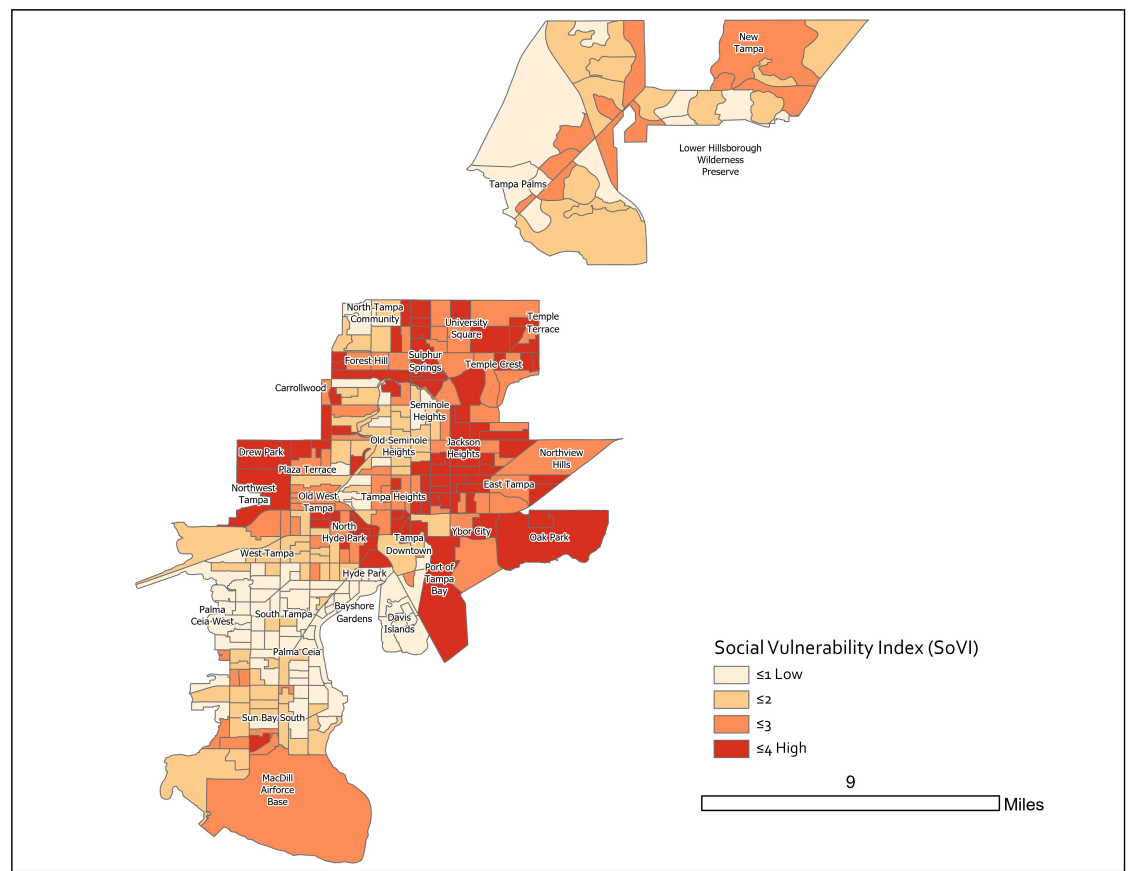

Fig. 5: Map of social vulnerability index, adapted from Wakhungu et al. (2021) 474 percentile of the frequency of valves' occurrence in the pareto frontier. The 475 number of times a valve occurred in the pareto frontier is counted as a fre- 


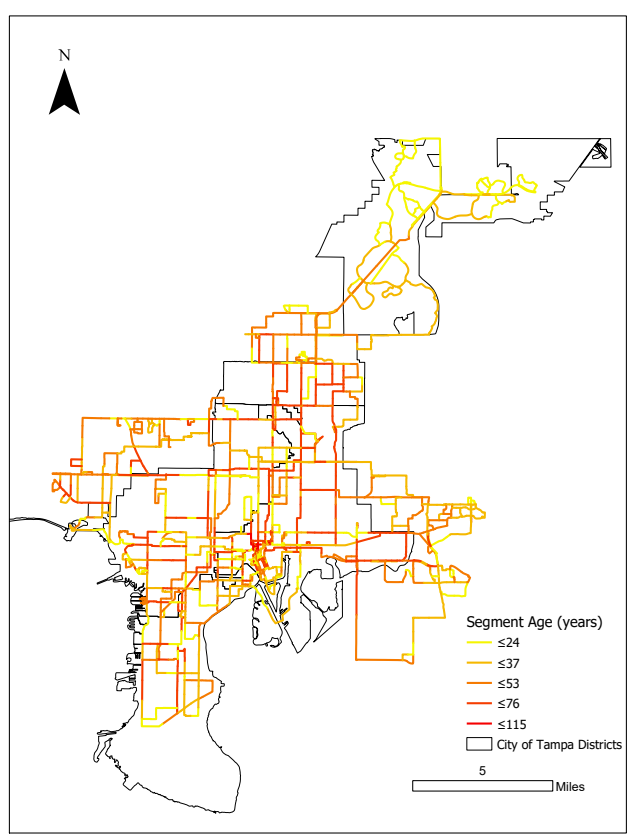

Fig. 6: Segment age mapped by color, age is used as a surrogate for the likelihood of failure

quency to provide an indication of criticality (relatively low or high compared to other valves selected by the model). The frequencies that valves appear in the solution set (or pareto frontier) from formulation 1, formulation 2 and formulation 3 are mapped in Figure 7. The deterministic model (formulation 1) selected 251 unique valves, the model accounting for uncertainties (formulation 2) selected 247 unique valves, and the model accounting for uncertainties and likelihood of failure (formulation 3) selected 278 unique valves. However, the results from formulation 2 had higher frequencies of valves occurring in the solution set because of the uncertainties accounted for. The results from Formulations 1 and 2 had more valves in the 75th and 100th percentile as shown in Figures 7a and Figure 7b, whereas formulation 3 diversifies the selected valves, reducing their frequencies in the solution set (as can be seen in Figure 7c). This is because the constraints added in formulation 3 emphasize 

512 compared at varying percentiles (as shown in Figure 8). 
4.3 Differences Between the Formulations

Both formulations 1 and 3 identified critical valves in the Northeast (New Tampa) and East Tampa, but the selected individual valves varied. These locations are selected by the model because they bridge between the reservoir and the periphery of the WDN. The variation in the individual valves is likely due to the likelihood of segment failure that is accounted for in formulation 3. In addition, there are more valves selected by the deterministic model (formulation 1) in East Tampa. When uncertainties and likelihood of failure are included (i.e., formulation 3) they are not selected, as shown in Figure 8c. This may be because of more newly installed pipes (as shown in Figure 6) and pipe replacement projects (FDOT, 2020; Water, 2018) that contribute to a lower likelihood of failure in East Tampa. Additionally, social vulnerability is relatively low in East Tampa in comparison with locations such as Ybor or North Tampa; meaning, there is lower uncertainty associated with the social vulnerability of segments in East Tampa, leading the model to select less valves in East Tampa when uncertainties are accounted for. Although the models selected many common valves (shown in Figure 8d), the deterministic model selects more valves in proximity to those common valves. This is likely due to the additional constraints (for the likelihood of failure) in formulation 3 limiting the selected valves in a given location. More importantly, the models can vary in the level of criticality assigned to selected valves. For example, formulation 3 selects valves in the Lake Leto area in the 25th percentile (Figure 8a), but formulation 1 selects valves from that location at the higher percentiles. In other words, the Lake Leto area is less critical when the likelihood of failure and uncertainty in flow volumes and social vulnerability are accounted for. This is an example of how including uncertainty and the likelihood of failure in the model provide more complete information for 
decision-makers. Additionally, including uncertainty and the likelihood of failure reduces the number of critical valves identified as the percentile (of the frequency of valves occurring in the solution set) increases. This is because formulation 3 includes additional constraints (i.e., based on their likelihood of failure), thereby eliminating solutions (i.e., sets of valves) that were identified by the exact method. The reduced number of critical valves identified at the higher percentiles makes the model results more readily considered by decision-makers. Another advantage of formulation 3 is that it allows valves to be identified as critical (even if at a lower percentile of frequency) where they otherwise may not have been selected at all. For example, if criticality is determined by the likelihood of failure alone, there would be less valves selected in the recently developed Northeast of the city (or the New Tampa location), but because the likelihood of failure is integrated within the model there are still valves selected with high frequency in the Northeast of the city. This is due to high values of reachability and flow volume of segments in the Northeast.

\subsection{Potential Implications of the Optimization Models}

This study applied multi-objective optimization to identify critical isolation valves in a real WDN. The optimization models were solvable within reasonable time and provided results that are useful to utilities. In particular the models made it easier to identify valves as critical that may have been missed using single objectives. The optimization model formulations allow decisionmakers to account for multiple facets of valve criticality. In particular, including social vulnerability in the optimization formulation identifies communities that may suffer unduly from an extended lack of water. If valves in these communities are operable, unsupplied demand to vulnerable customers may 


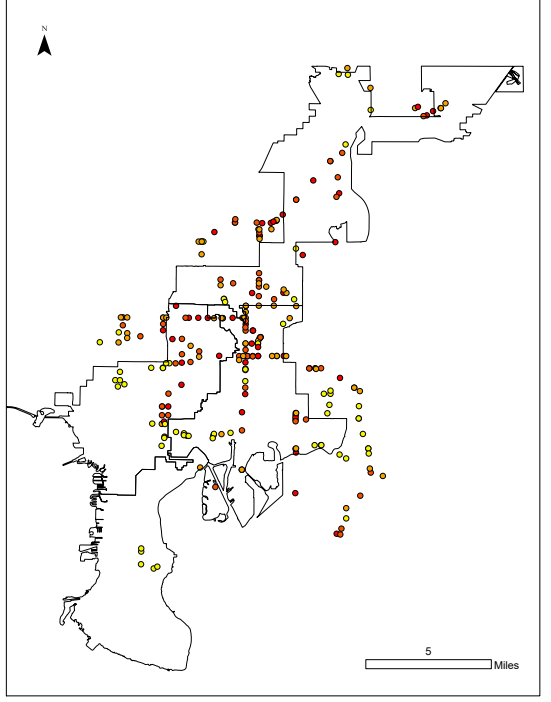

(a) Formulation 1

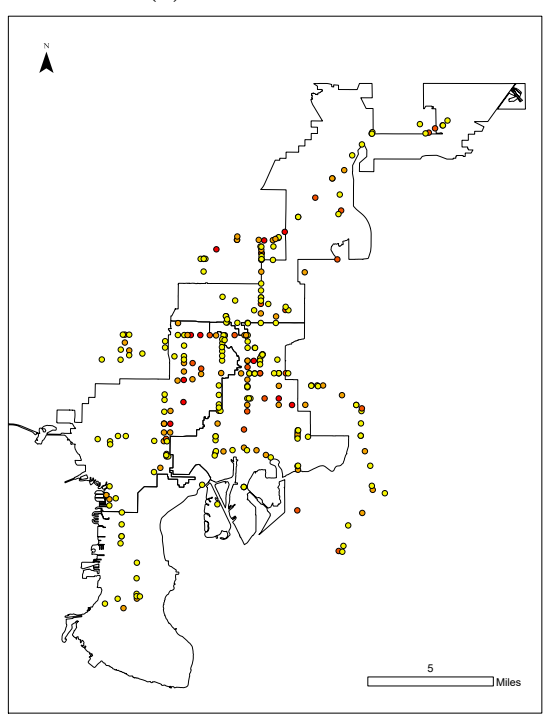

(c) Formulation 3

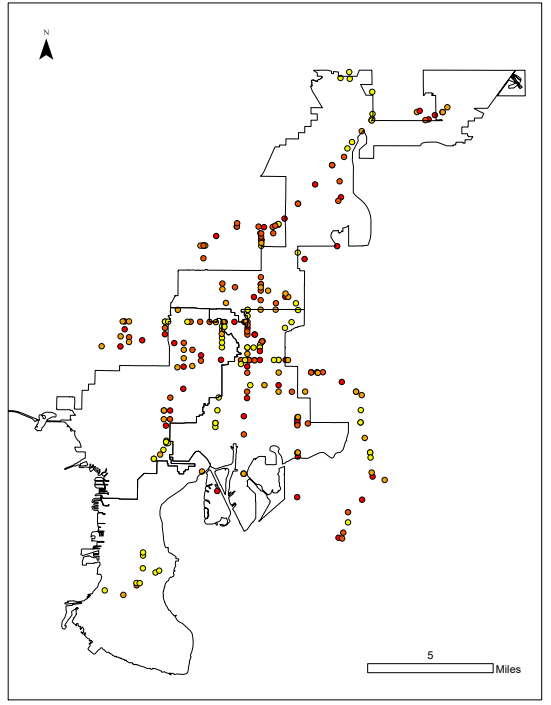

(b) Formulation 2

\section{Frequency}

- 25th Percentile

50th Percentile

- 75th Percentile

- 25th Percentile

Fig. 7: Maps of identified valves using the three formulations, where Formulation 1 is deterministic, Formulation 2 accounts for uncertainty, and Formulation 3 accounts for uncertainty and likelihood of failure 


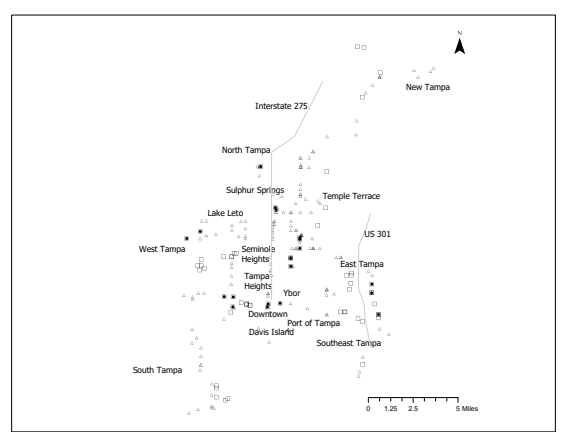

(a) 25th percentile

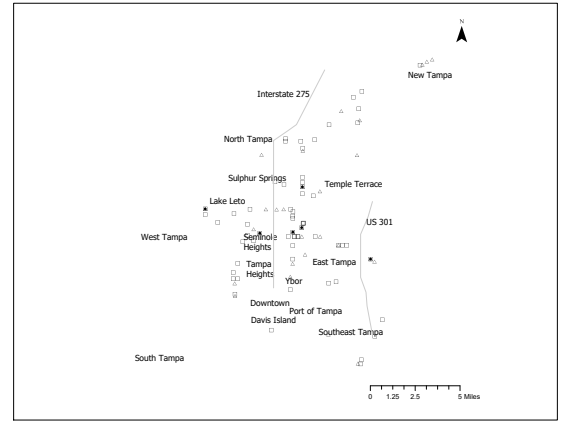

(c) 75 th percentile

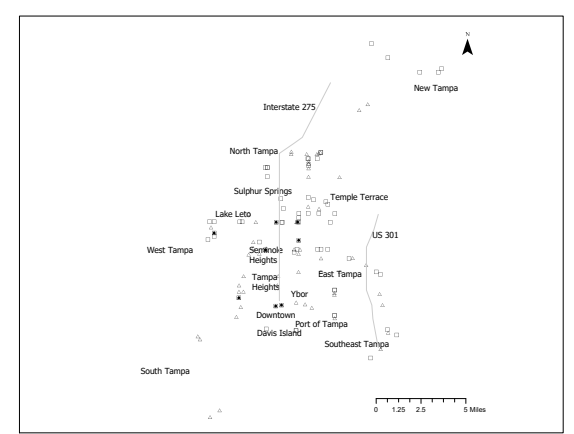

(b) 50 th percentile

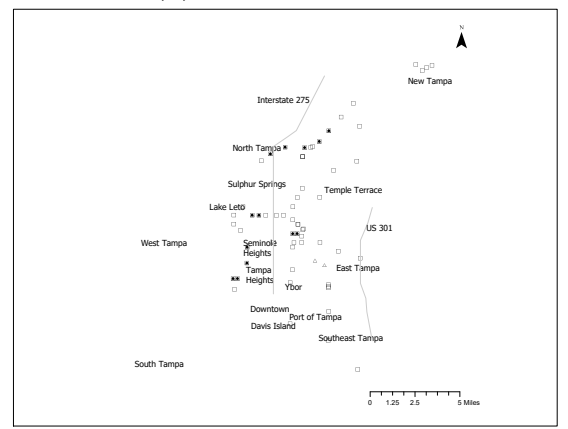

(d) 100th percentile

\section{$\square$ Deterministic $\triangle$ Robust-Probabilistic • Intersect}

Fig. 8: Maps of valves identified by formulations 1 and 3 at different percentile for the frequency of occurrence in the solution set

566 be reduced. Formulation 2 in particular allows decision-makers to take uncer-

${ }_{567}$ tainties of segment attributes into account. Even more useful, formulation 3 ${ }_{568}$ prioritized the selection of valves based on the likelihood of failure. Ultimately, 569 the formulation 3 diversifies the identified valves while also selects less unique 570 valves at higher percentiles of criticality. Obtaining the common valves iden571 tified by both formulations 1 and 3 may help hone in on highly critical valves. ${ }_{572}$ This is because valves selected by both models are not only critical but also 573 connect the segments with high likelihood of failure and high uncertainties as574 sociated with flow volume and social vulnerability. Because the models result 
in many repeated valves, the frequencies of valves occurring in the solution sets can be used to rank the selected valves or divide them into varying levels of priority. This can offer utilities a powerful alternative to the method used in traditional asset management, such as multiplying the consequence of failure and likelihood of failure (Ispass, 2008; Pollard, 2016; Park et al., 2010), which limits the identified critical components (i.e., does not identify all of them) (Abdel-Mottaleb et al., 2019).

However, there remain limitations to this study. The consequence of failure scores used by utilities may have more than three variables factored in. In that case, when using the current models, some variables would be combined (i.e., weighted together), but the results would be more diverse than weighing all the variables as a single objective (i.e., traditional score of the consequence of failure) assigned to segments. Though social vulnerability is used as an objective function, it may be substituted with a lifeline facility indicator (e.g., number of hospitals and schools in a census block, number of shelters) — which may be more useful to utilities for analyzing the impact of inoperable valves after disturbances (Emrich, 2005; Borden et al., 2007). In this study, likelihood of failure was reduced to a surrogate based on pipe age, whereas in reality it is also affected by the length of pipe in a segment, soil conditions, pipe material, among many other things. However, this does not impact the methodology because the values from the function for the likelihood of failure that a utility uses for their asset management can be input to the model the same way.

\section{Conclusion}

This study formulated models for identifying critical isolation valves in WDN.

The study proposed the use of a Gomory-Hu tree to represent how segment isolation can ultimately propagate within a WDN. A major contribution of the 
study is applying graph theory to simplify the combinatorial problem of multiple valves affecting segments (because of multiple possible flow paths between segments). This can help identify critical valves without running many scenarios, including combinations of operable and inoperable valves. The Gomory-Hu tree may also be useful in the failure analysis of other flow-based infrastructure networks because it helps to ascertain the logical implications of component failure in a network. Further, the study evaluated the critical valves not only based on hydraulics, but also on social vulnerability. The identified critical valves differed when social vulnerability was included as an objective function in the optimization procedure - suggesting that social indicators (be they demographic or related to lifeline facilities) should be included in infrastructure criticality analyses. Another advance the study made is accounting for both uncertainties (in water flow and social vulnerability) and the likelihood of failure of segments when identifying critical valves; the model accounting for uncertainties and likelihood of failure was compared with the deterministic model. Results showed that the formulation accounting for uncertainties selects more valves, but the formulation accounting for uncertainties and likelihood of failure narrows the selection down due to additional constraints when accounting for the likelihoods of failure. All optimization models were solvable within a reasonable time and different formulations provide additional insights that are useful to utilities. The multi-objective optimization model makes it easier to identify critical valves that may have been missed using single objectives or traditional assessment management methods. Future work can address the limitations of the current study by using the actual likelihood of failure from utilities instead of a surrogate based on pipe age. Additionally, including a lifeline facility indicator related to segments in the objective functions can help utilities determine critical valves, especially under extreme disturbances. 
634 Not Applicable

${ }_{635}$ Consent to Participate

${ }_{636}$ Not Applicable

${ }_{637}$ Consent to Publish

${ }_{638}$ Not Applicable

Authors Contributions

${ }_{640}$ Conceptualization: [N. Abdel-Mottaleb, Q. Zhang], Methodology: [N.Abdel${ }_{641}$ Mottaleb, Q. Zhang, P. Saghand, H. Charkhgard, M. Wakhungu, E. C. Wells ], ${ }_{642}$ Formal analysis and investigation: [N. Abdel-Mottaleb, Q. Zhang, P. Saghand, ${ }_{643}$ M. Wakhungu], Writing - original draft preparation: [N. Abdel-Mottaleb, P. ${ }_{644}$ Saghand, M. Wakhungu]; Writing - review and editing: [N. Abdel-Mottaleb, ${ }_{645}$ Q. Zhang, P. Saghand, H. Charkhgard, M. Wakhungu, E. C. Wells], Fund${ }_{646}$ ing acquisition: [Q. Zhang, E. C. Wells], Resources: [Q. Zhang, C. Wells, H. ${ }_{647}$ Charkhgard], Supervision: [Q. Zhang, H. Charkhgard, E. C. Wells]. 
${ }^{4} 48 \quad$ Funding

649 This material is based upon work supported by the National Science Founda650 tion under Grant Number 1638301. Any opinions, findings, and conclusions or 651 recommendations expressed in this material are those of the authors and do ${ }_{652}$ not necessarily reflect the views of the National Science Foundation.

${ }_{653}$ Competing Interests

${ }_{654}$ The authors declare no conflicts of interest.

Availability of data and material 663 ysis.

The water distribution network for the city of Tampa is confidential due to security reasons. The code to obtain the Gomory-Hu Tree and to run the multi-objective optimization are available upon request from the corresponding author. All software used to obtain data and analyze results is cited in the paper. The commercial software Arcpro, WaterGEMS, and Gurobi are used for generating maps, hydraulic simulations and optimization, respectively. Open source software python and the networkx package are used for network anal-

\section{References}

Abdel-Mottaleb, N., Ghasemi Saghand, P., Charkhgard, H., and Zhang, Q. (2019). An exact multiobjective optimization approach for evaluating water distribution infrastructure criticality and geospatial interdependence. Water Resources Research, 55(7):5255-5276.

Abdel-Mottaleb, N. and Walski, T. (2020). Identifying vulnerable and critical water distribution segments. American Society of Civil Engineers. 
Abdel-Mottaleb, N. and Walski, T. (2021). Evaluating segment and valve importance and vulnerability. Journal of Water Resources Planning and Management, 147(5):04021020.

Bentley Systems (2020). Bentley WaterGEMS.

Boland, N., Charkhgard, H., and Savelsbergh, M. (2017). The quadrant shrinking method: A simple and efficient algorithm for solving tri-objective integer programs. European Journal of Operational Research, 260(3):873-885.

Borden, K. A., Schmidtlein, M. C., Emrich, C. T., Piegorsch, W. W., Cutter, S. L., et al. (2007). Vulnerability of us cities to environmental hazards. Journal of Homeland Security and Emergency Management, 4(2):1-23.

Chavez, M. A. (2013). Desert in the springs: Ethnography of a food desert.

Creaco, E. and Haidar, H. (2019). Multiobjective optimization of control valve installation and dma creation for reducing leakage in water distribution networks. Journal of Water Resources Planning and Management, 145(10):04019046.

Cutter, S. L., Boruff, B. J., and Shirley, W. L. (2003). Social vulnerability to environmental hazards. Social science quarterly, 84(2):242-261.

Dächert, K. and Klamroth, K. (2015). A linear bound on the number of scalarizations needed to solve discrete tricriteria optimization problems. Journal of Global Optimization, 61(4):643-676.

Diao, K., Sitzenfrei, R., and Rauch, W. (2019). The impacts of spatially variable demand patterns on water distribution system design and operation. Water, 11(3):567.

Emrich, C. T. (2005). Social vulnerability in US metropolitan areas: improvements in hazard vulnerability assessment. $\mathrm{PhD}$ thesis, Citeseer.

FDOT (2020). Completed project finder.

Giustolisi, O. (2020). Water distribution network reliability assessment and isolation valve system. Journal of Water Resources Planning and Manage- 
ment, 146(1):04019064.

Giustolisi, O. and Savic, D. (2010). Identification of segments and optimal isolation valve system design in water distribution networks. Urban Water Journal, 7(1):1-15.

He, X. and Yuan, Y. (2019). A framework of identifying critical water distribution pipelines from recovery resilience. Water Resources Management, 33(11):3691-3706.

Hernandez, E. and Ormsbee, L. (2021). Segment-based assessment of consequences of failure on water distribution systems. Journal of Water Resources Planning and Management, 147(4):04021009.

Ispass, A. (2008). A practical approach to implementing asset management. In 2008 WEF Utility Management Conference.

Jun, H., Loganathan, G., Deb, A., Grayman, W., and Snyder, J. (2007). Valve distribution and impact analysis in water distribution systems. Journal of Environmental Engineering, 133(8):790-799.

Liu, H., Walski, T., Fu, G., and Zhang, C. (2017). Failure impact analysis of isolation valves in a water distribution network. Journal of Water Resources Planning and Management, 143(7):04017019.

Mahmoud, H. A., Kapelan, Z., and Savić, D. (2018). Real-time operational response methodology for reducing failure impacts in water distribution systems. Journal of Water Resources Planning and Management, 144(7):04018029.

Mays, L. W. et al. (2000). Water distribution systems handbook. McGraw-Hill,. Paez, D., Filion, Y., Castro-Gama, M., Quintiliani, C., Santopietro, S., Sweetapple, C., Meng, F., Farmani, R., Fu, G., Butler, D., et al. (2020). Battle of postdisaster response and restoration. Journal of Water Resources Planning and Management, 146(8):04020067. 
Park, S., Vega, R., Choto, Z., and Grewe, M. (2010). Risk-based asset prioritization of water transmission/distribution pipes for the city of tampa. Florida Water Resources, December, pages 22-28.

Pollard, S. (2016). Risk management for water and wastewater utilities. Iwa publishing.

Saghand, P. G., Charkhgard, H., and Kwon, C. (2019). A branch-and-bound algorithm for a class of mixed integer linear maximum multiplicative programs: A bi-objective optimization approach. Computers $\& 3$ Operations Research, 101:263-274.

Santonastaso, G., Di Nardo, A., and Creaco, E. (2019). Dual topology for partitioning of water distribution networks considering actual valve locations. Urban Water Journal, 16(7):469-479.

Shuang, Q., Liu, H. J., and Porse, E. (2019). Review of the quantitative resilience methods in water distribution networks. Water, 11(6):1189.

Shuang, Q., Zhang, M., and Yuan, Y. (2014). Performance and reliability analysis of water distribution systems under cascading failures and the identification of crucial pipes. PloS one, 9(2).

Wakhungu, M. J. (2020). An ethnography of wash infrastructures and governance in sulphur springs, florida.

Walski, T. M. (2001). The wrong paradigm — why water distribution optimization doesn't work. Journal of Water Resources Planning and Management, 127(4):203-205.

Water, T. B. (2018). Capital improvements program fiscal years 2019-2028.

WaterWorld (2014). Case study: Ok water district updates distribution systems with isolation valves.

Zischg, J., Rauch, W., and Sitzenfrei, R. (2018). Morphogenesis of urban water distribution networks: a spatiotemporal planning approach for cost-efficient and reliable supply. Entropy, 20(9):708. 
754 Zischg, J., Reyes-Silva, J. D., Klinkhamer, C., Krueger, E., Krebs, P., Rao, P. S. C., and Sitzenfrei, R. (2019). Complex network analysis of water distribution systems in their dual representation using isolation valve information. In World Environmental and Water Resources Congress, pages 484-497. ASCE Pittsburgh, Pennsylvania. 
Figures

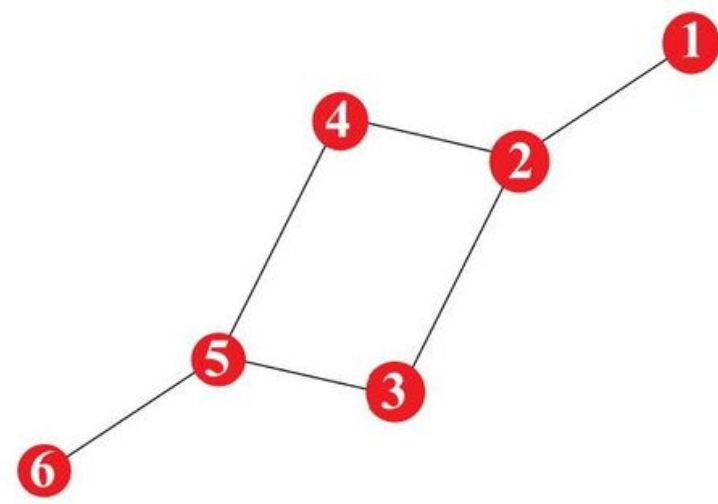

(a) segment-valve representation of a small example network

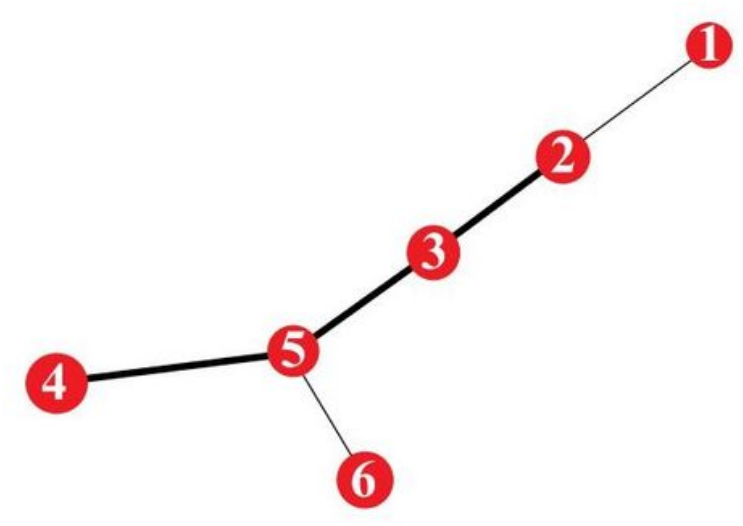

(b) Gomory-Hu tree of small example, where edges that represent more than one valve are bolded

\section{Figure 1}

Segment-valve represenation and Gomory-Hu tree of a network example 


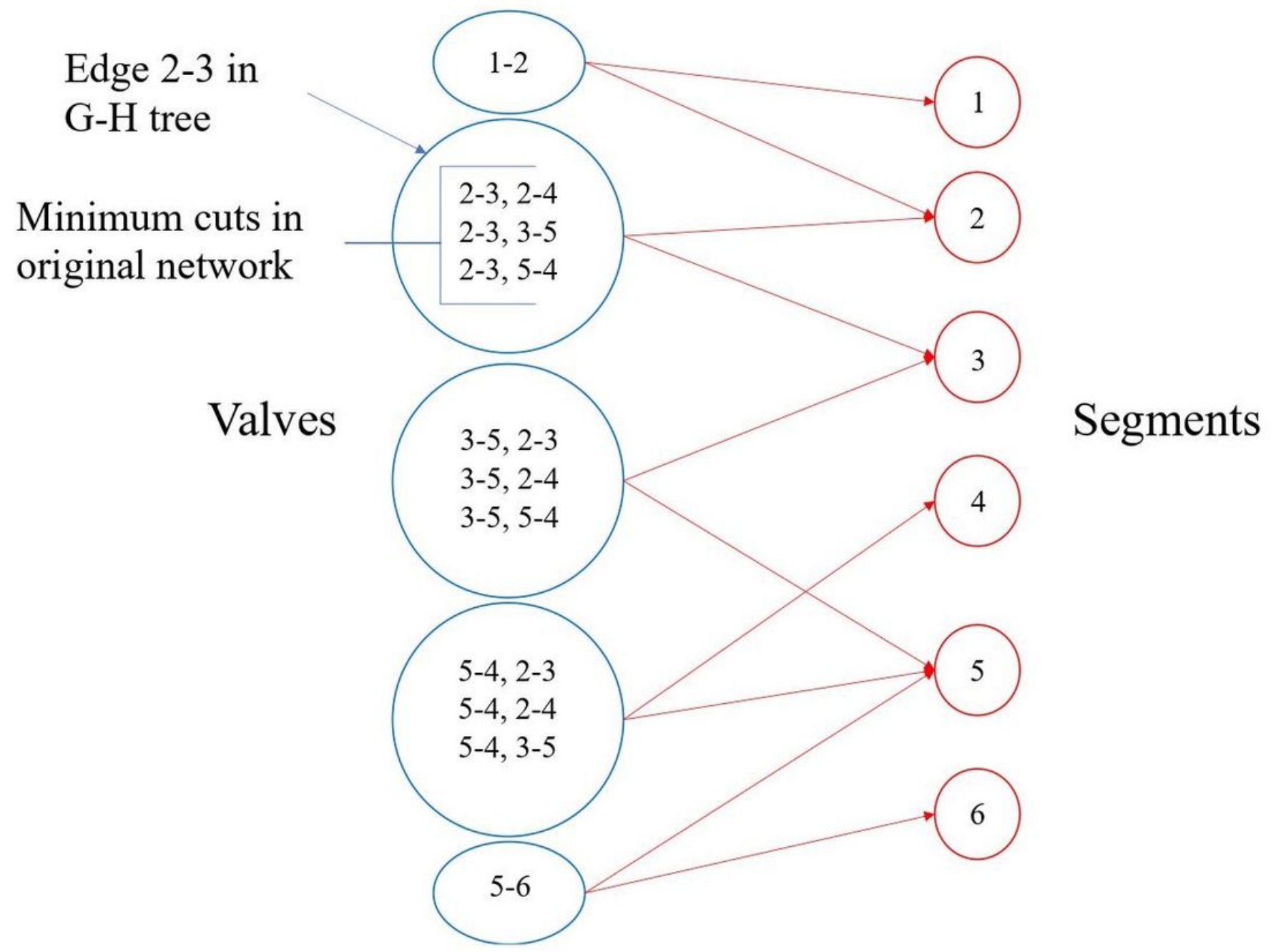

Figure 2

representation of the logical network, where valves that were represented by edges are modeled as nodes and segments are also modeled as nodes, and the edges between valves and segments represent logical implications Note: The designations employed and the presentation of the material on this map do not imply the expression of any opinion whatsoever on the part of Research Square concerning the legal status of any country, territory, city or area or of its authorities, or concerning the delimitation of its frontiers or boundaries. This map has been provided by the authors. 


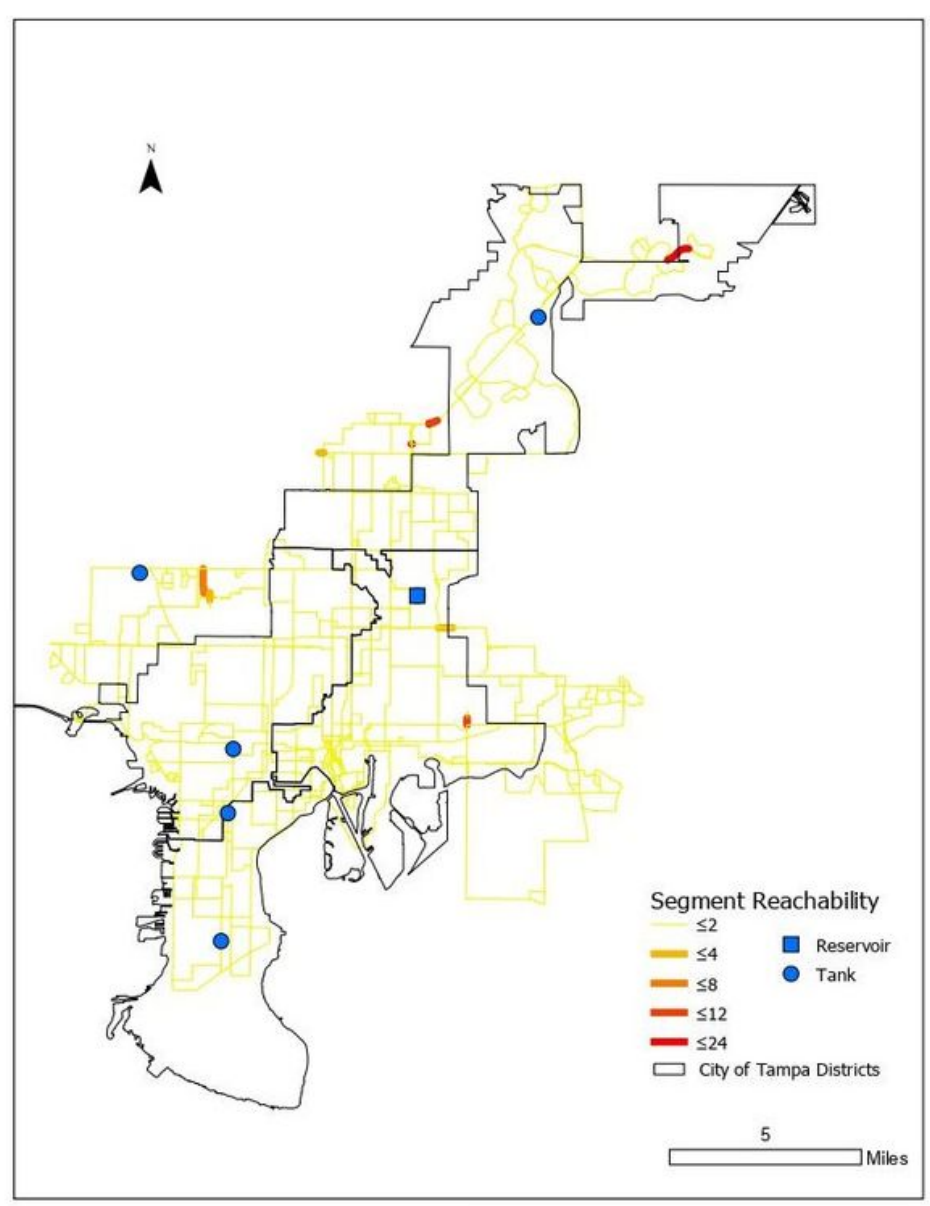

(a) Map of segment reachability values

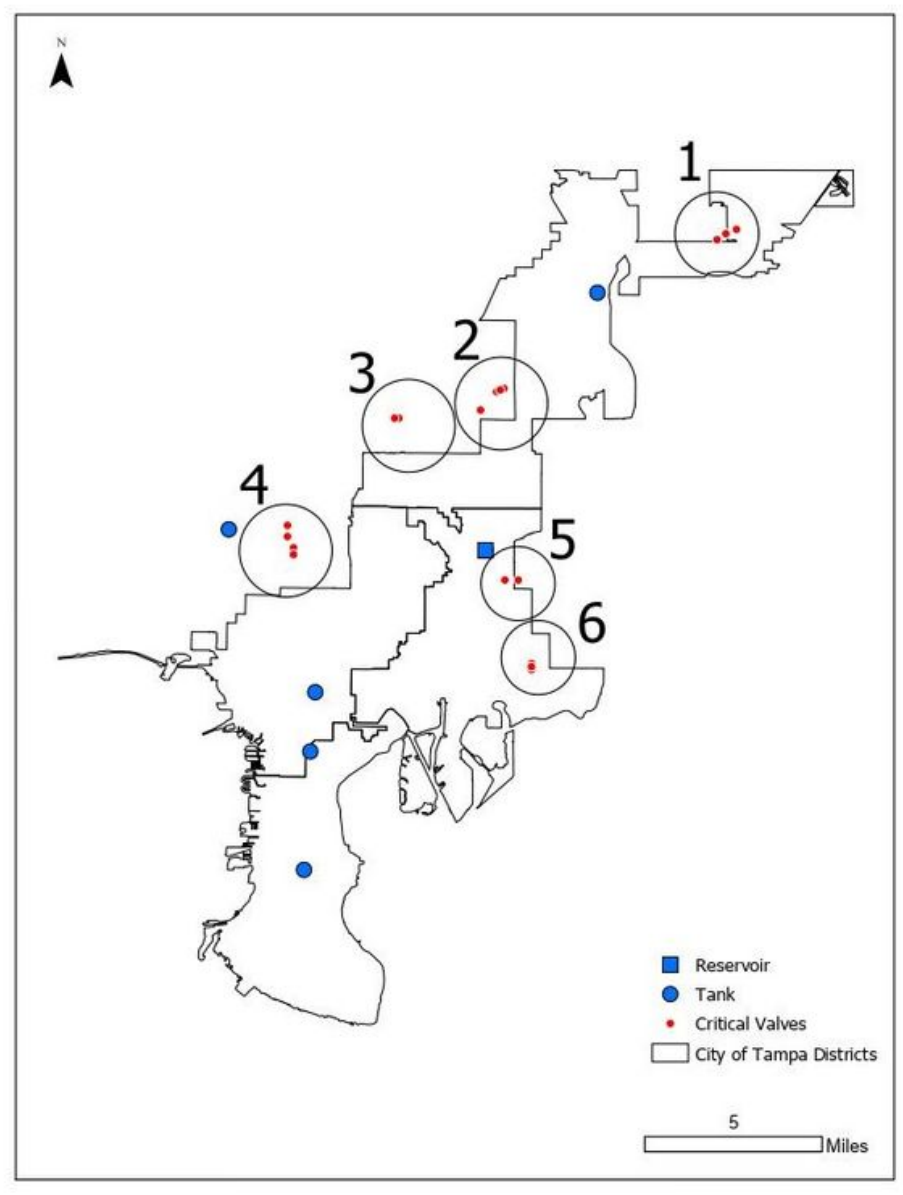

(b) Map of critical valves

\section{Figure 3}

Segment reachability values and the subsequently identified critical valves Note: The designations employed and the presentation of the material on this map do not imply the expression of any opinion whatsoever on the part of Research Square concerning the legal status of any country, territory, city or area or of its authorities, or concerning the delimitation of its frontiers or boundaries. This map has been provided by the authors. 


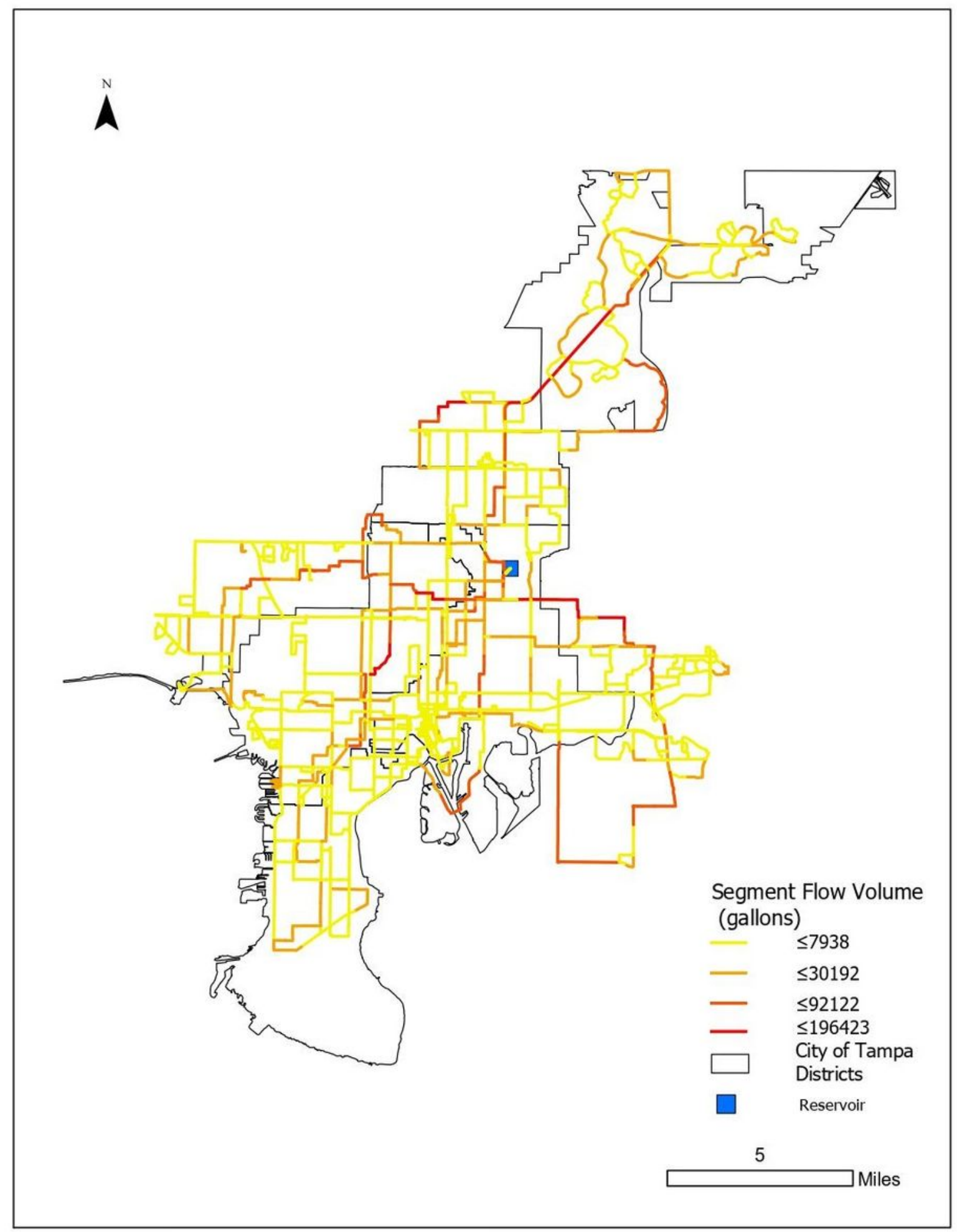

Figure 4

Map of segment flow volume Note: The designations employed and the presentation of the material on this map do not imply the expression of any opinion whatsoever on the part of Research Square concerning the legal status of any country, territory, city or area or of its authorities, or concerning the delimitation of its frontiers or boundaries. This map has been provided by the authors. 


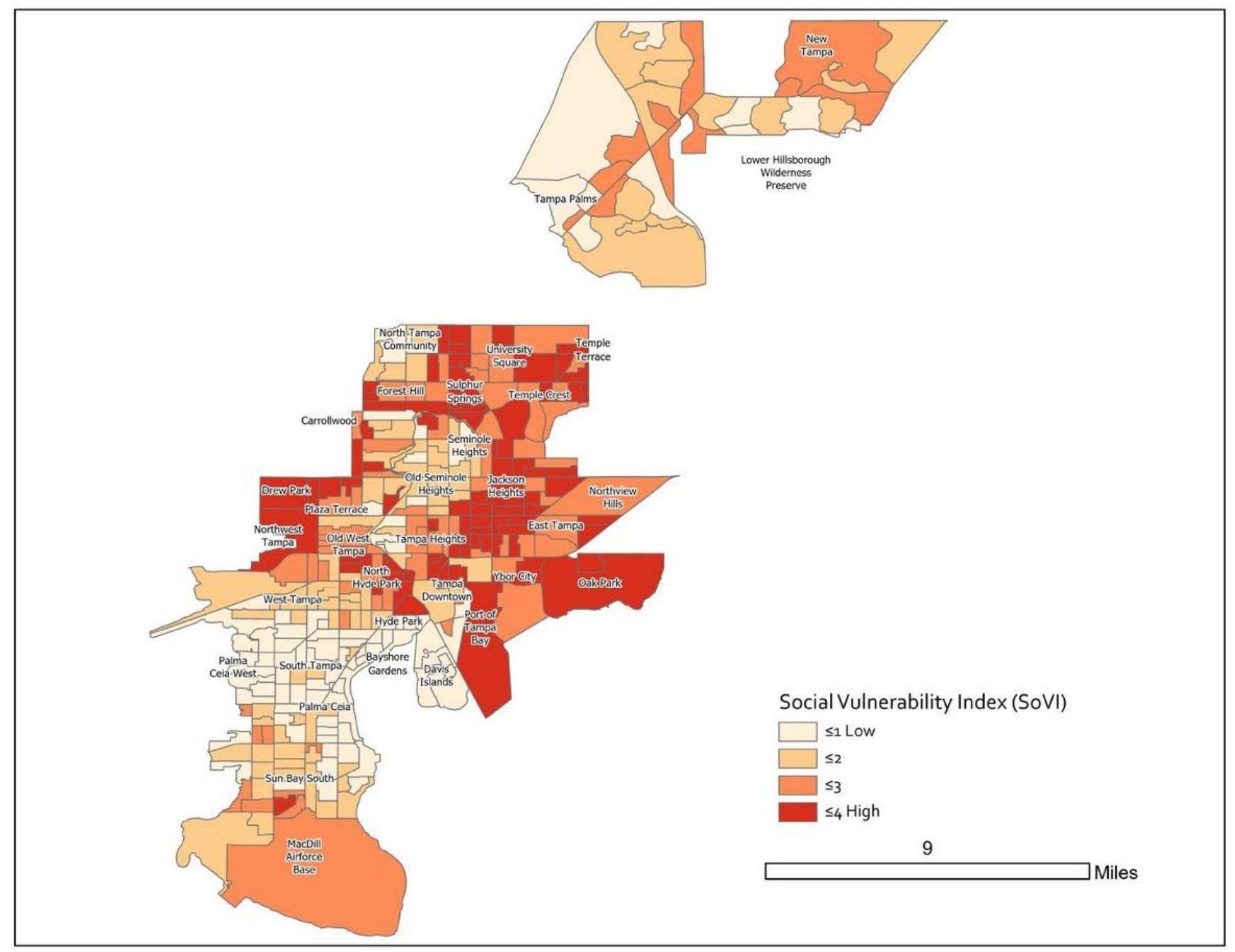

Figure 5

Map of social vulnerability index, adapted from Wakhungu et al. (2021) Note: The designations employed and the presentation of the material on this map do not imply the expression of any opinion whatsoever on the part of Research Square concerning the legal status of any country, territory, city or area or of its authorities, or concerning the delimitation of its frontiers or boundaries. This map has been provided by the authors. 


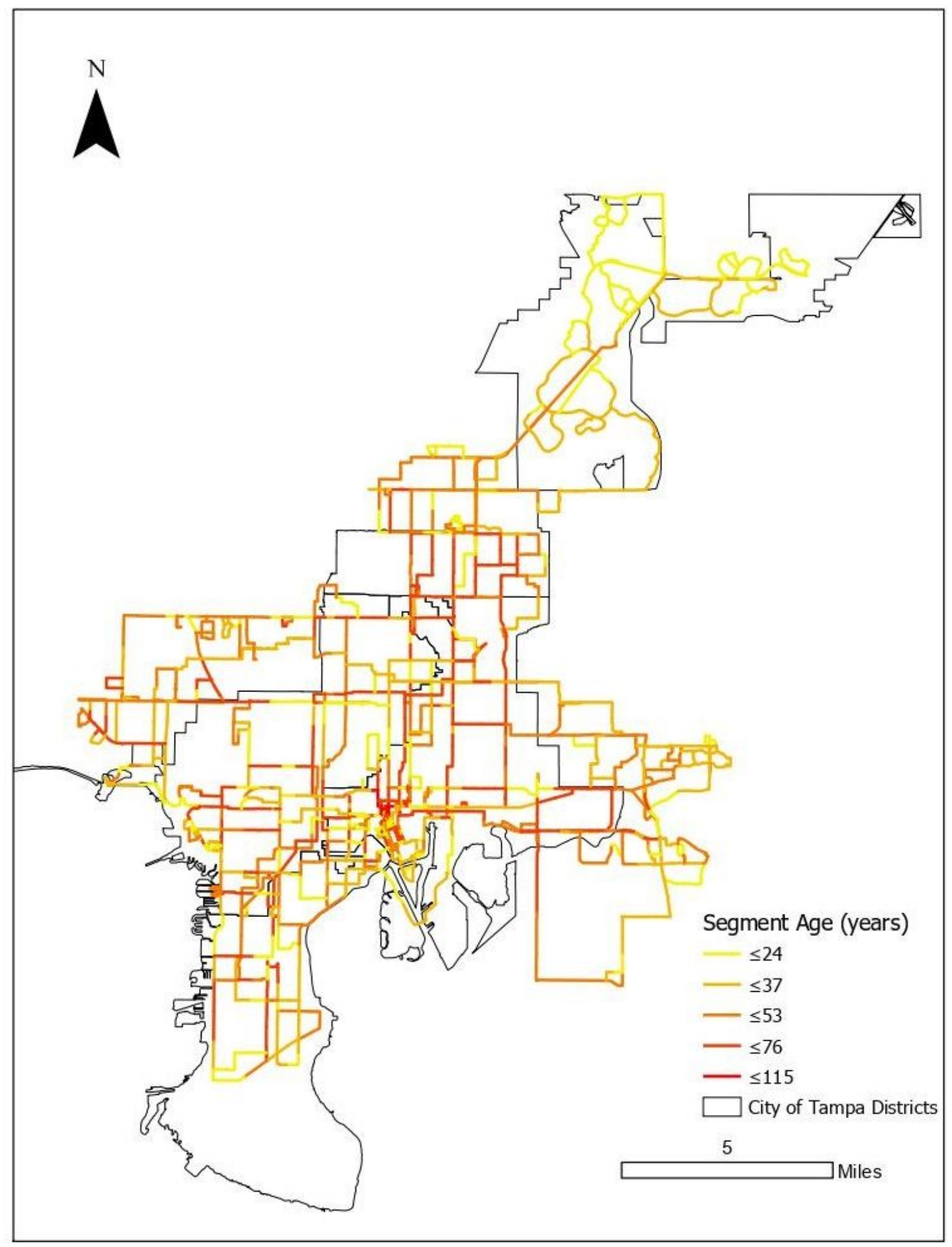

\section{Figure 6}

Segment age mapped by color, age is used as a surrogate for the likelihood of failure Note: The designations employed and the presentation of the material on this map do not imply the expression of any opinion whatsoever on the part of Research Square concerning the legal status of any country, territory, city or area or of its authorities, or concerning the delimitation of its frontiers or boundaries. This map has been provided by the authors. 


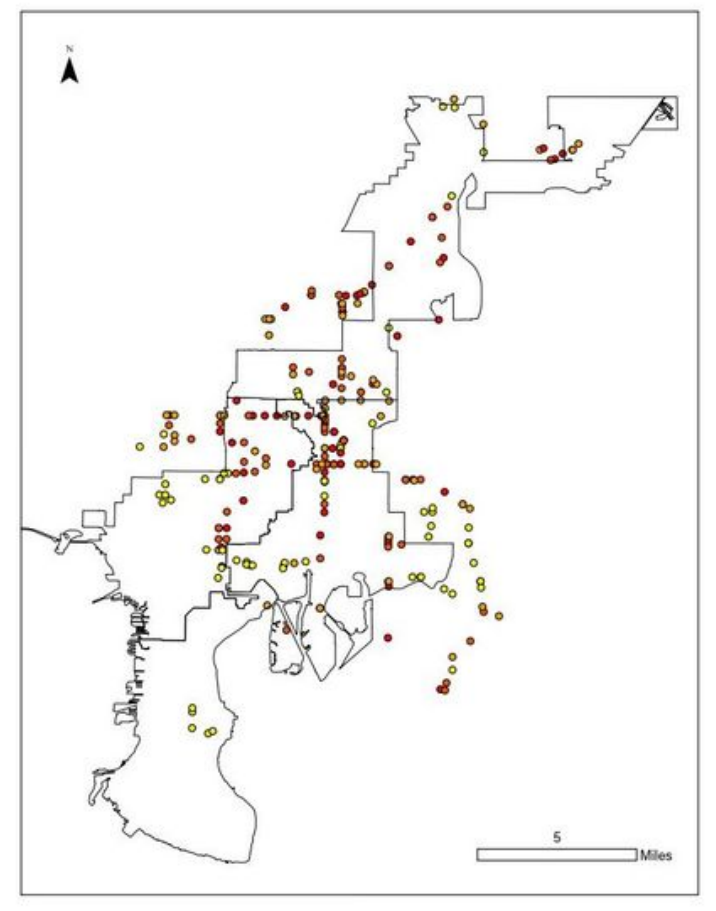

(a) Formulation 1

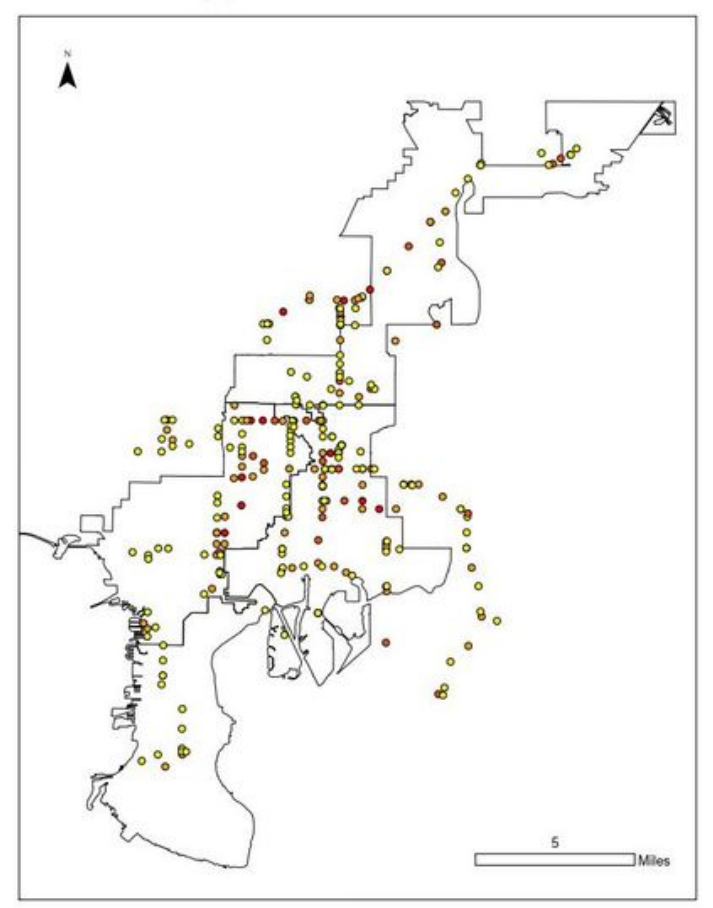

(c) Formulation 3

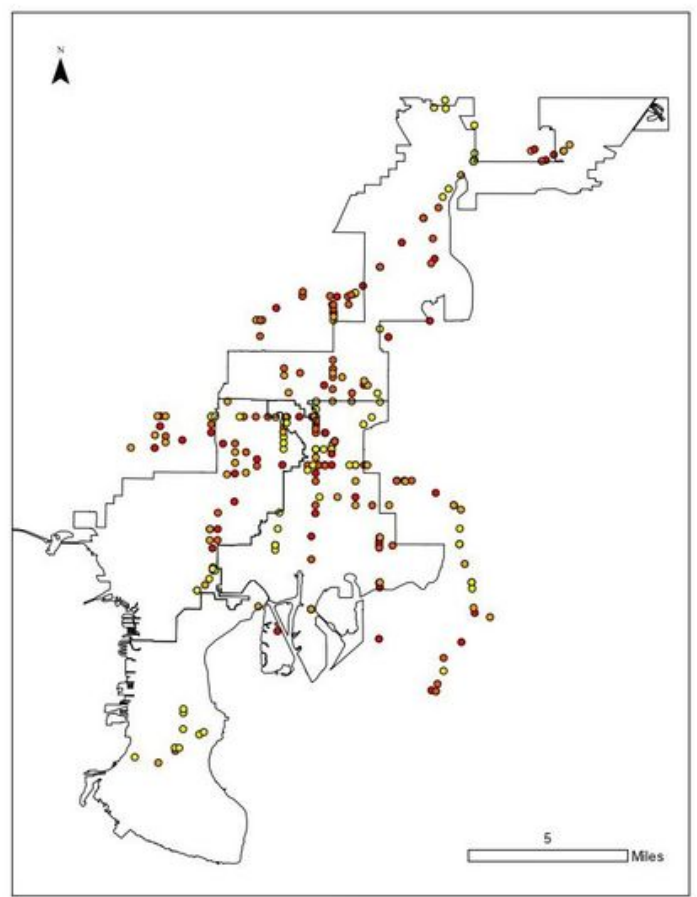

(b) Formulation 2

\section{Frequency}

- 25th Percentile

- 50th Percentile

- 75th Percentile

- 25th Percentile

\section{Figure 7}

Maps of identified valves using the three formulations, where Formulation 1 is deterministic, Formulation 2 accounts for uncertainty, and Formulation 3 accounts for uncertainty and likelihood of failure Note: The designations employed and the presentation of the material on this map do not imply the expression of any opinion whatsoever on the part of Research Square concerning the legal status of any country, 
territory, city or area or of its authorities, or concerning the delimitation of its frontiers or boundaries. This map has been provided by the authors.

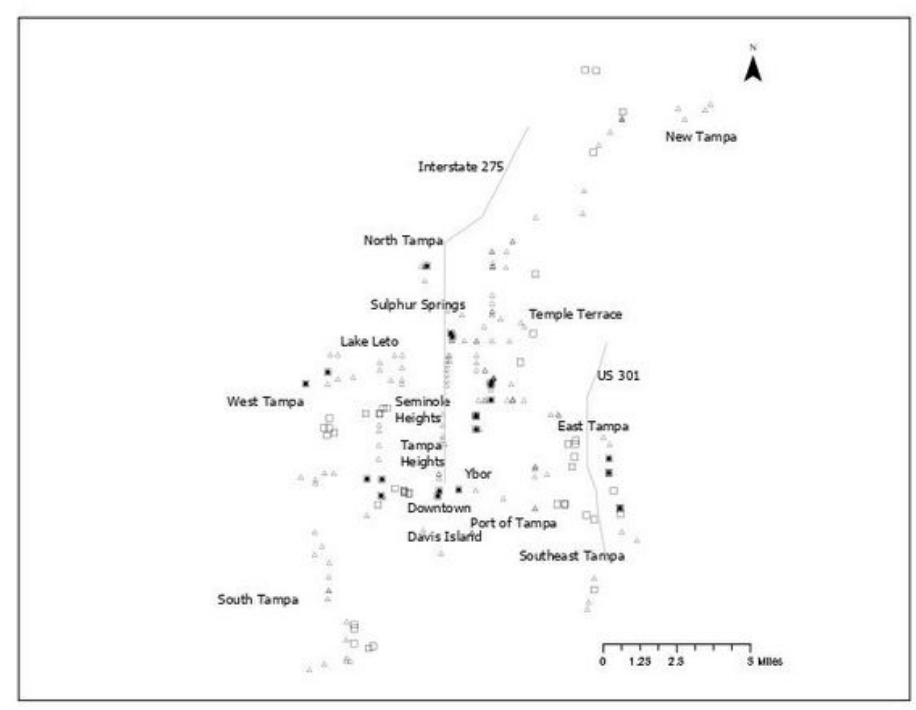

(a) 25th percentile

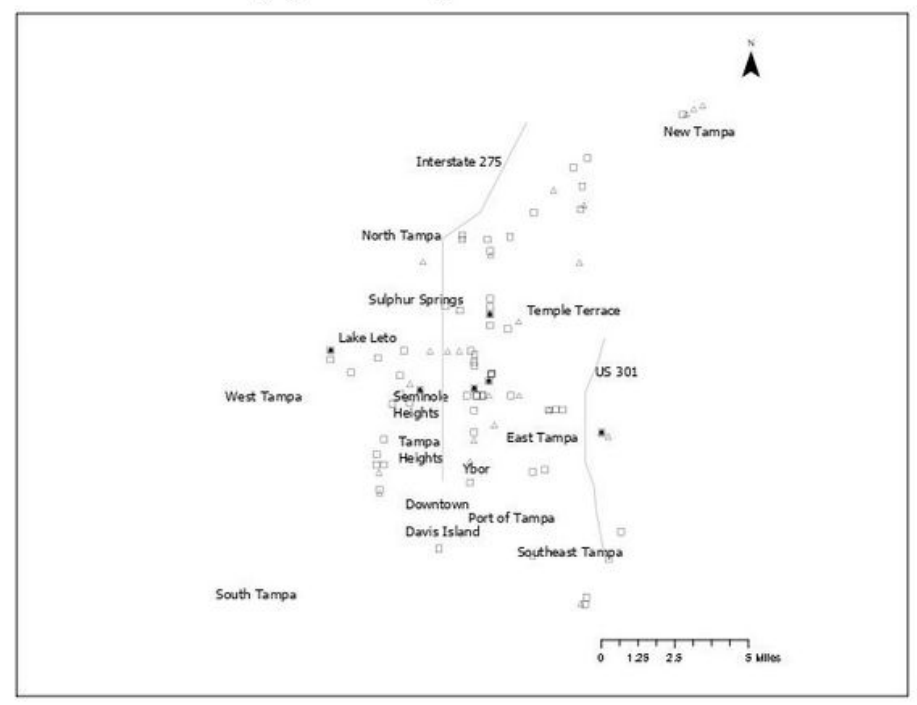

(c) 75 th percentile

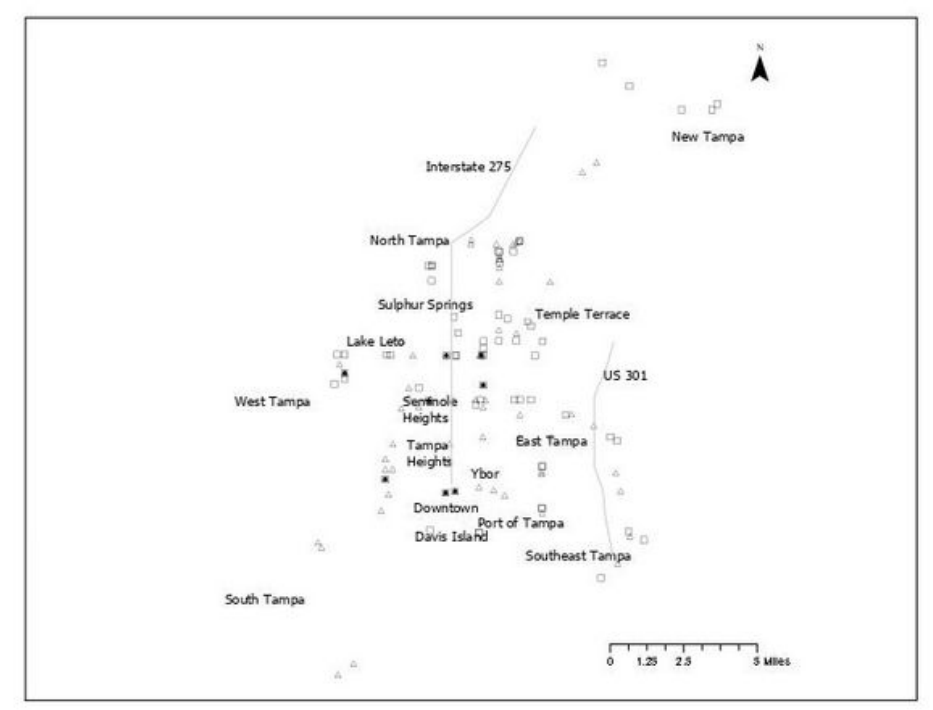

(b) 50th percentile

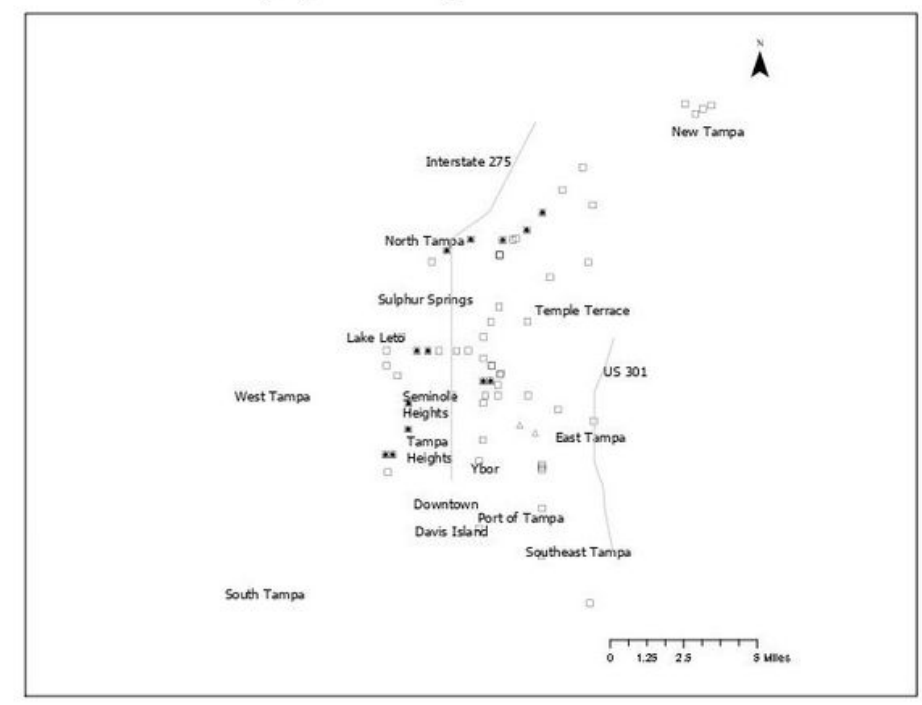

(d) 100th percentile

\section{Deterministic $\triangle$ Robust-Probabilistic $\bullet$ Intersect}

\section{Figure 8}

Maps of valves identified by formulations 1 and 3 at different percentile for the frequency of occurrence in the solution set Note: The designations employed and the presentation of the material on this map do not imply the expression of any opinion whatsoever on the part of Research Square concerning the legal status of any country, territory, city or area or of its authorities, or concerning the delimitation of its frontiers or boundaries. This map has been provided by the authors. 